\title{
NUCLEI OF PLANETARY NEBULAE
}

\section{IN THE PULSATION INSTABILITY STRIP}

\author{
W. Dziembowski \\ Copernicus Astronomical Center \\ Polish Academy of Sciences \\ Aleje Ujazdowskie 4 \\ 00-478 Warszawa \\ POLAND
}

\begin{abstract}
Pulsation properties of relevant stellar models are investigated with the linear nonadiabatic method. Short-period variability of FG sge is interpreted as a pulsation phenomenon.
\end{abstract}

This investigation was carried out in connection with the discoveries of short-period variability in FG Sge. The star known to be a nucleus of a planetary nebula is a unique object in which evolutionary processes are taking place on the time scale of years. Its spectral type changed from $B 4$ in 1955 to $F 6$ in 1972 (Langer et al., 1974). In 1975 the spectral type was G2 according to Smolinski et al. (1976).

The variability of the star with a period of about 60 days was found by Papousek (1972) from his photometric observations made in 1971. The amplitudes were $0.45 \mathrm{mag}$ and $0.2 \mathrm{mag}$ in $\mathrm{B}$ and $\mathrm{V}$ colours, respectively. From her observations made in 1975, Acker (1977) found radial velocity variations with an amplitude of about $100 \mathrm{~km} / \mathrm{s}$ and a period of about 20 days. 
Such a period change was difficult to understand within the framework of the pulsation hypothesis which on the other hand seemed plausible in view of the spectral type of the star. Since possibly, 1. the non-linear pulsation development may be quite different in this object and in such stars as RR Lyrae or Cepheids; 2 . the period determination could be wrong, it appeared of interest to investigate pulsational properties of relevant stellar models.

Following Paczyński (1970) it was assumed that nuclei of planetary nebulae are double shell burning stars containing only small fraction of their mass in hydrogen and helium rich envelopes. Evolution of such objects is complicated by helium flashes giving rise to loops on the tracks in H-R diagram of large extend in $\log T_{e}$. The behaviour of FG Sge has been interpreted as a manifestation of this phenomenon (Paczyński, 1970; Sparks and Kutter, 1973; Christy-Sackmann, 1974).

For double shell burning models there is the following relation between mass of the core, M, and luminosity, $I$,

$L / L O=59250 \times M / M O-30950$, for $M / M_{O} \geqslant 0.6$ and Population $I$ composition ( $\mathrm{x}=0.7, \mathrm{z}=0.03)$, (c.f. Paczý́ski, 1970).

This relation remains approximately valid also during the flashes. Since the mass contained in the envelopes of our models was negligible, the above formula was used as the mass luminosity relation for the models with mass greater than $0.6 \mathrm{M} \odot$ and consequently L greater than $4600 \mathrm{~L} \oplus$. For lower luminosites, calculations were carried out for $M=0.52,0.56,0.6 \mathrm{M} \mathcal{O}$. Thermal equilibrium in the unperturbed envelope models was assumed. Opacity was calculated with the Stellingwerf (1975) formula. Only linear nonadiabatic code was used to study pulsation properties of the models.

Positions in H-R diagram of the blue edges of the pulsation instability strip extension to this high luminosities were found. Effective temperature at the blue edge does not decrease monotonically with the luminosity increase as it is the case for classical pulsating variables. Instead, the minimum of $\log \mathrm{T}_{e}$ of about 3.74 is reached at $\log L / L \mathcal{O}=3.4$ and a further luminosity increase results in a shift of the blue edge toward higher effective temperatures. For $\log \mathrm{L} / \dot{\mathrm{L}} O=4.4(\mathrm{M} / \mathrm{M} O=0.9) \log \mathrm{T}_{\mathrm{e}}=3.85$ at the blue edge. This somewhat unexpected phenomenon is a consequence of the fact that at such high luminosities there is very little dissipation in the radiation dominated region below the HeII ionization zone. 
Periods at the blue edges vary from 11.6 - 12.9 days (depending on mass) for $\log \mathrm{L} / \mathrm{L} O=3.0$ to 270 days for $\log \mathrm{L} / \mathrm{L} O=4.45$ and $\mathrm{M} / \mathrm{M} O=1.0$. In the latter case, the pulsation period is of the same order as the time scale for crossing the instability strip during the flash, so that the assumption of equilibrium for unperturbed envelope was not justified. Development of more or less periodic pulsations cannot be expected at that high luminosities.

In all models considered pulsations are truly nonadiabatic. The adiabatic value of period is quite different from the true linear value. At $\log \mathrm{L} / \mathrm{L} \mathcal{O}=3.8$ the former is about two times larger. Variations of period within the instability strip are very nonhomologous. In the vicinity of the blue edge the period is a slow function of the effective temperature and for $\log \mathrm{L} / \mathrm{L} \sigma=3.9$ it decreases with the temperature decrease. Strength of the pulsational instability is usually expressed by the excitation rate $\eta=4 \pi \operatorname{Re}(\omega) /$ $/ \operatorname{Im}(\boldsymbol{\omega})$ if the temporal dependence of perturbations is $\exp (\boldsymbol{\omega} t)$. Thus, if $\eta$ is small it is equal to the fractional pulsation energy increase during one period. For models of the clasical pulsating stars $\eta$ is of the order of few hundredths while for the present models it reaches values two orders of magnitude larger. Therefore, a very violent development of the instability may be expected once the star moves toward the middle of the instability strip. It is likely that this results in an ejection of outer parts of the envelope.

Identifying the spectroscropic period of 20 days with the pulsation period one finds that luminosity of FG Sge must be less than 1600 Lo . This luminosity may be too low for a star to develop helium flashes (c.f. Paczýski, 1974).

If the 60 day period found from photometry is identified with the pulsation period, then the implied luminosity is about $6300 \mathrm{~L} 0$ and $M$ is about $0.63 \mathrm{M} O$. This value of mass is between two estimates based on the time scale of evolution made by Christy-Sackmann and Despain (1974) who got $0.77 \mathrm{M} O$ and by Paczynski (1975) who got $0.55 \mathrm{M} O$.

The blue edge for the sequence with $M=0.63 \mathrm{M} O$ is at $\log$ $T_{e}=3.79$. In the year of Papousek's observations, the spectral type of FG Sge was F5 as derived from scanner observations or F8 as implied by colours. The former type which is more reliable implies $\log T_{e}=$ 3.83 , the latter $\log \mathrm{T}_{\mathrm{e}}=3.79$. Bearing in mind various uncertainties involved, one may conclude that the coincidence of the 
effective temperature determinations with the effective temperature at the blue edge supports the pulsation hypothesis.

Four years later the star had spectral type G2 which corresponds to $\log \mathrm{T}_{\mathrm{e}}=3.70$. At such effective temperature, the excitation rate is well above one. Thus, if the present interpretation is correct, the development of instability could lead to dramatic changes in the stellar envelope.

It would be of great interest to have some other records of the FG Sge variability in the period 1971 - 1975 as well as to have data on current behaviour of the star. Discovery of new variable nuclei of planetary nebulae having late $F$ and early $G$ spectral types would be also of great importance.

Acknowledgements I thank Professor B.Paczyński for suggesting to me the subject of this research as well as for many helpful conversations. It is a pleasure to acknowledge that the computation reported in this paper were made with the PDP $11 / 45$ computer donated to the Copernicus Astronomical Center by the US National Academy of Sciences, following the initiative and through the action of Dr. R. C. O Dell and Mr. A. M. Baer.

\section{References}

Acker,A. 1977, Astron. Astrophys. in press

Christy-Sackmann, I.-J., Despain,K.H. 1974, Astrophys. J. 189, 523

Langer,G.E., Kraft,R.P., Anderson,K.S. 1974, Astrophys. J. 189, 509

Paczyński,B. 1970, Acta Astron. 20, 47

Paczyński,B. 1974, Astrophys. J. 192,483

Paczyński,B. 1975, Astrophys. J. 202, 558

Papousek,J. 1972, Scripta Fac. Sci. Nat. Ujep Brunensis. Physica 2, 2,115

Smoliński,J., Clinenhaga,J.L., Kipper,T. 1976, Publ. Astr. Soc. Pac. 86,67

Sparks,w., Kutter,G. 1973, Astrophys. J. Letters 183, L121

stellingwerf, R.F. 1975, Astrophys. J. 195, 441 